\title{
A THEORY OF POGGENDORFF ILLUSION
}

\author{
CHUN CHIANG
}

Institute of Physics, Academia Sinica, Taipei

\begin{abstract}
Poggendorf illusion is attributed to be the consequence of the spurious enhancement of receptive field excitation, the lateral inhibition, the optical image blurring, the perceptual image distribution, the variation of reference standards, and depth processing. The perceptual image distribution and the variation of reference standards are newly introduced for explaining the illusion. Rotation effect on illusion is explained on the basis that the physical horizontal and vertical are adopted in the physiological system for reference, thus it is more difficult to induce the illusion when the orientation is in either horizontal or vertical. The equation to incorporate above effects is
\end{abstract}

$\mathrm{I}=(\mathrm{A}+\mathrm{B}|\sin 2 \phi|)\left[\mathrm{K}_{\mathrm{g}}-\mathrm{K}_{\mathrm{l}}+\mathrm{K}_{\mathrm{b}}+\mathrm{K}_{\mathrm{p}}+\mathrm{K}_{\mathrm{r}}{ }^{\prime}\left(1-\mathrm{e}^{-2 / 5}\right)+\mathrm{K}_{\mathrm{d}}\right] \mathrm{d} \cot \theta+\mathrm{K} \sin 4 \phi$

where $K, A, B$ and $\tau$ are constants, $\lambda$ is contrast, $K_{b}, K_{1}, K_{b}, K_{p}, K_{r}$ and $K_{d}$ represent the effect of the spurious enhancements, lateral inhibition, image blurring, the perceptual image distribution, the variation of reference standards and the depth processing respectively; $d$ is the width of the 2 parallel lines; $\theta$ is the angle between the inclined line and the parallel lines, $\phi$ is the angle between the inclined line and the horizontal. $\left(A+B\left|\sin ^{2} 2 \phi\right|\right) \beta\left|\sin ^{2} \phi\right|$ represents the easiness to induce the illusion with respect to rotation, and $\sin 4 \phi$ represents the illusion when there is no inducing parallel lines.

In a previous paper (Chiang, 1968), the Poggendorff illusion was explained on the basis of the optical image blurring in the retina, and other illusions of the crossing lines type have in turn been explained by the Poggendorff effect.

The result of optical blurring is that when two objects are presented to the eyes, the optical image of the objects on the retina do not quite correspond to the physical shapes of the objects, and the lines $\mathrm{AC}$ and BC (see Fig. 1) converge before they really reach the intersection points. Thus lines $\mathrm{BC}$ and $\mathrm{EH}$ seem to be misaligned. The contribution of the optical blurring to the illusion has been experimentally verified by Coren (1969). However, many authors (for example Cumming, 1968; Pressey \& den Heyer, 1968; Restle, 1969, Farné, 1970) have pointed out that the optical blurring effect alone is insufficient to explain all the feature of the illusion. Von Békésy (1967), Ganz (1966) and Blakemore, Carpenter and Georgeson
(1970) have proposed lateral inhibition as a source for the illusion; Coren (1970) has shown that when possible sources of inhibitory interactions are removed, the illusion still remains. Gillam (1971) and Gregory (1963) have used the perspective theory to explain the illusion. Weintraub and Krantz (1971) have cited evidence that the stability of vertical and horizontal orientations, and assimilation towards the vertical or horizontal also contribute to

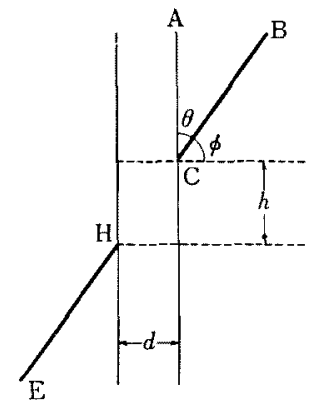

Fra. 1. A conventional Poggendorff illusion. 
the illusions. Recently Walker (1973) assumes that the spurious enhancement of receptive field excitations near the intersection of the image lines on the retina contributes to the cortical determination of the geometry of two-dimensional figures. Chiang (1973a) has further used the concept of perceptual image distribution, and variation of reference standards, for the explanation of the Poggendorff illusion. The purpose of this paper is to explore the Poggendorff illusion in more detail and to propose an equation to explain quantitatively the available data.

\section{Perceptual Image Distribution}

When physical objects are presented to the eyes, optical images are formed on the retina. These optical images are then projected onto the perceptual space via nerve impulses, and these impulses are also interpreted as physical objects located at points in physical space. The positions of the objects in physical space and the positions of the objects in perceptual space maintain certain projection relationships, which may not be unique. Fig. 2 shows the projection relationship between physical and perceptual space. The horizontal axis represents the position and the vertical axis represents the probability of finding or perceiving the objects at that position. The projection function between physical and perceptual space is assumed to be normal distribution. Thus there is a finite probability of perceiving the objects in some position of perceptual space for which there are no physical objects at the corresponding positions in physical space; thus, the perceived position of the physical object is not unique, and fluctuates around the corresponding physical position.

If two objects are well separated as shown in Fig. 2a, the perceptual images of
(A)

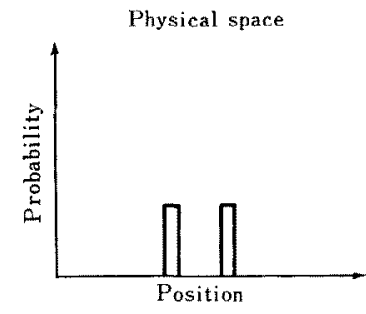

(B)

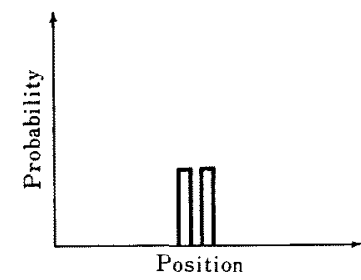

Perception space
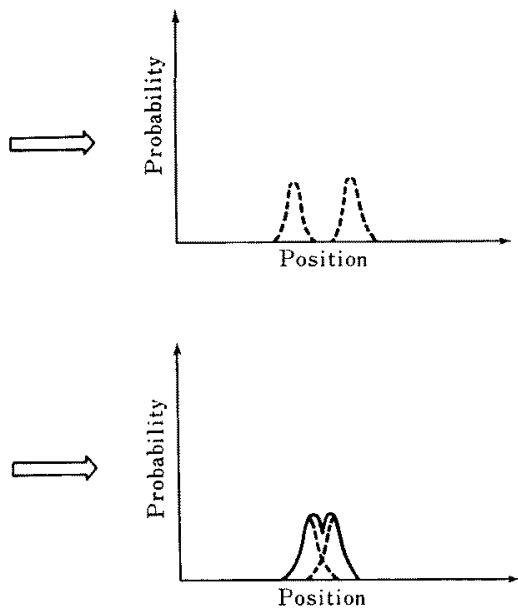

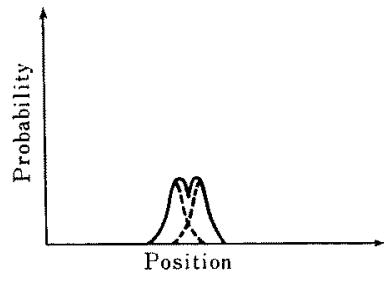

FIG. 2. The projection relationship between the physical space and the perception space. An object fixed in the physical space may be perceived to locate in different position in the perception space.

(a) When the separation between two objects is large, there is no interaction in the perception space.

(b) When the separation between two objects is small, there is interaction in the perception space such that the maximum probability positions shift to each other. 
these two objects show no mutual interaction; however, if two objects are close enough as shown in Fig. 2b, then these two perceptual images are coupled to each other. With the superposition of two individual probability distribution, the position of each maximum probability for perceiving the object shifts toward each other. Thus, the perceived distance between the two objects decreases. Many illusions may be explained by this principle. In this paper, we confine our attention to the Poggendorff illusion.

\section{Factors Influencing the Poggendorff Illusion}

Spurious enhancement of receptive field excitations. Walker (1973) assumes that the spurious enhancement of receptive field excitations near the intersection point of the image lines on the retina contributes to the cortical determination of the location of the intersection lines. This enhancement causes a shift of the lines, thus producing the illusion. The amount of illusion resulting from this factor can calculate to be, in terms of the displacement of two noncollinear lines, as (Walker, 1973)

$$
\mathrm{I}_{\mathrm{s}}=\left[\frac{1-\frac{8 \mathrm{KL}}{3 \pi \mathrm{d}} \sec \theta\left(\mathrm{F} \cot \frac{\theta}{2} \cos \frac{\theta}{2}-\mathrm{F}^{\prime} \tan \frac{\theta}{2} \sin \frac{\theta}{2}\right)}{1+\mathrm{K} \sec \theta \csc \theta\left(\mathrm{f} \cot \frac{\theta}{2} \cos \frac{\theta}{2}-\mathrm{f}^{\prime} \tan \frac{\theta}{2} \sin \frac{\theta}{2}\right)}\right] \mathrm{d} \cot \theta,
$$

where $L$ is the length of the transversal lines, $d$ is the width of the two parallel lines and $K, F, F^{\prime}, f, f^{\prime}$ are constant, and $\theta$ is the angle between the transversal and the parallel lines. For convenience, we

$$
\mathrm{K}_{\mathrm{s}}(\theta)=1-\frac{1-\frac{8 \mathrm{KL}}{3 \pi \mathrm{d}} \sec \theta\left(\mathrm{F} \cot \frac{\theta}{2} \cos \frac{\theta}{2}-\mathrm{F}^{\prime} \tan \frac{\theta}{2} \sin \frac{\theta}{2}\right)}{\mathrm{I}+\mathrm{K} \sec \theta \csc \theta\left(\mathrm{f} \cot \frac{\theta}{2} \cos \frac{\theta}{2}-\mathrm{f}^{\prime} \tan \frac{\theta}{2} \sin \frac{\theta}{2}\right)}
$$

From Walker's treatment (1973), we can see that $\mathrm{K}_{\mathrm{s}}(\theta)$ varies little with $\theta$, thus, for all practical purpose, we can regard it as constant. Walker (1973) has shown that Eq. (1) fits to his experimental data (variation of $\theta$ ) very closely, also, Weintraub and Krantz (1971) have also shown that the illusion varies linearly with $\cot \theta$.

Lateral inhibition. Blakemore, Carpenter and Georgeson (1970), von Békésy (1967) and Ganz (1966) have proposed lateral inhibition as the source for illusion; due to the neural interaction, the location of two neighboring lines may shift. Ganz supposes the lateral inhibition effects arise at the retina level, while Blakemore, Carpenter and Georgeson suppose these to occur at the cortical level. However, Walker (1973) has pointed out that the can write Eq. (1) as

$$
\mathbf{I}_{\mathrm{s}}=\mathbf{K}_{\mathrm{s}}(\theta) \mathrm{d} \cot \theta,
$$

where $\mathrm{K}_{\mathrm{s}}(\theta)$ is

proposed lateral inhibition should produce a reversed illusion. If this is the case, the amount of illusion produced by lateral inhibition could similarly be calculated by Walker's method (1973) to be

$$
\mathrm{I}_{1}=-\mathbf{K}_{\mathrm{l}}(\theta) \mathbf{d} \cot \theta,
$$

where $K_{1}(\theta)$ represents a similar equation to Eq. (3). The negative sign indicates that the illusion is in the reversed direction.

Optical image blurring. Chiang (1968) has proposed optical image blurring on the retina as the source of the illusion. The magnitude of illusion, $I_{b}$, due to the enlargement of an acute angle from optical blurring can also be calculated to be

$$
\mathrm{I}_{\mathrm{b}}=\mathbf{K}_{\mathrm{b}}(\theta) \mathrm{d} \cot \theta,
$$


where $\mathrm{K}_{\mathrm{b}}(\theta)$ is of the same form as $\mathrm{Eq}$. (3). The elimination of this factor has been shown by Coren (1969) and Imai (1973) to decrease the amount of illusion.

Perceptual image distribution (Chiang, 1973

a). From this principle, line $\mathrm{AC}$ and line $\mathrm{BC}$ will interact when they approach close enough (it is not necessary for them to intersect). The interaction will be such that the lines $\mathrm{AC}$ and $\mathrm{BC}$ will shift toward each other near the intersection point $C$. However, there is no interaction between point $A$ and point $B$, because the distance between them is large. Thus the acute angle looks perceptually larger, and for this kind of illusion, $I_{p}$ would also be

$$
\mathrm{I}_{\mathrm{p}}=\mathrm{K}_{\mathrm{p}}(\theta) \mathrm{d} \cot \theta,
$$

where $\mathrm{K}_{\mathrm{p}}$ is of a form as Eq. (3). This effect is operative even if line $\mathrm{AC}$ and line $\mathrm{BC}$ do not intersect. From this principle, it predicts that the illusion will decrease gradually with the separation between the transversal and parallel lines. This is indeed shown to be true by Imai (1973).

Variation of reference standards (Chiang, 1973a). One judges the orientation of the lines by some standards stored in memory. However, when the lines are blocked by some other strong signals such as the two parallel lines in Poggendorff figure, the original orientation standards are overshadowed and the two parallel lines are used as orientation standard. Line BE intersects the two vertical lines at point $\mathrm{C}$ and point $\mathrm{H}$, and since point $\mathrm{C}$ is physically higher than point $\mathrm{H}$, the notion of point $\mathrm{C}$ being higher than point $\mathrm{H}$ is "associated" and "transfered" to the notion that line $\mathrm{EH}$ and line $\mathrm{BC}$ are misaligned with point $\mathrm{C}$ being shifted upwards and point $\mathrm{H}$ downwards. This "association" and "transfer" process is similar to that involved in "association" of a meaning to a symbol or a language. The magnitude of misjudgment $I_{r}$ is proportional to the vertical distance $h$ between point $\mathrm{H}$ and point $\mathrm{C}$, namely,

$$
\mathrm{I}_{\mathrm{r}}=\mathrm{K}_{\mathrm{r}} \mathrm{h}=\mathrm{K}_{\mathrm{r}} \mathrm{d} \cot \theta,
$$

where $K_{r}$ is a constant. Pressey and Sweeney (1972) have argued that in judging the collinearity of the transversal lines, the subject projects a series of obliques; because of "differential attention", the shorter obliques are weighted more heavily than the longer obliques, thus the transversal assimilates to the parallel lines and gives rise to the illusion. That argument implies that if we change the attention, this process can be reversed. This does not seem to be true, thus we prefer to omit the argument that it is due to the differential attention which gives rise to the illusion, rather, we simply argue that it is due to the fact that the point $\mathrm{C}$ locates above the point $\mathrm{H}$ along the parallel lines, which erroneously gives rise to the illusion according to Eq. (7).

From this principle, it predicts that any factor which could influence the extent of the reference standard will effect the illusion. Thus, the illusion will depend on the thickness and the contrast of the parallel lines, both of which can effect the extent of the blocking and reference. Imai (1973) has indeed shown that thickening the parallel increases the illusion. Weintraub and Krantz (1971) have shown that lowering the contrast decreases the illusion. Thus $\mathrm{K}_{\mathrm{r}}$ is a function of thickness and contrast. From the data of Weintraub and Krantz, we can write $K_{r}=$ $\mathbf{K}_{\mathbf{r}}{ }^{\prime}\left(1-\mathrm{e}^{-\tau / \lambda}\right)$, where $\mathbf{K}_{\mathbf{r}}{ }^{\prime}$ and $\tau$ are constant and $\lambda$ is the contrast.

Depth processing. Gillam (1971) has used the depth processing theory to explain the illusion. Under ordinary condition, this contribution may not be very much, however, with a strong three-dimensional cue, the contribution may be significant. The illusion due to this factor may also approximate to be

$$
\mathrm{I}_{\mathrm{d}}=\mathrm{K}_{\mathrm{d}} \mathrm{d} \cot \theta .
$$

Assuming linear independence of the above factors, the total amount of illusion would be 


$$
\begin{aligned}
\mathrm{I}= & \mathrm{I}_{\mathrm{s}}+\mathrm{I}_{\mathbf{l}}+\mathrm{I}_{\mathrm{b}}+\mathrm{I}_{\mathrm{p}}+\mathrm{I}_{\mathrm{r}}+\mathrm{I}_{\mathrm{d}} \\
= & {\left[\mathrm{K}_{\mathrm{s}}-\mathrm{K}_{1}+\mathrm{K}_{\mathrm{b}}+\mathrm{K}_{\mathrm{p}}+\mathrm{K}_{\mathrm{r}}{ }^{\prime}\left(1-\mathrm{e}^{-\lambda / \tau}\right)\right.} \\
& \left.+\mathrm{K}_{\mathrm{d}}\right] \mathrm{d} \cot \theta .
\end{aligned}
$$

This equation shows that the total illusion is approximately proportional to $\cot \theta$, similar to earlier data (Walker, 1973; Weintraub \& Krantz, 1971). The explanation and some experimental evidence for the contribution of the individual component of this equation have been discussed in previous sections. However, due to the fact that available data is incomplete, the estimation of these components is not yet possible.

\section{Negative Illusion}

Bouma and Andriessen (1970) have presented some interesting data on the orientation of line segments influenced by other lines. Their data show that when the angle between the inducing and the induced lines are small, the direction of the illusion is reversed. This is contradictory to other data (Velinsky, 1925; Weintraub \& Krantz, 1971); however, a closer look reveals that Bouma and Andriessen used a very short line segment. Using the principle of perceptual image distribution, it is possible to explain it as follows: If $\mathrm{BC}$ line in Fig. 1 is very short such that the distance between point $A$ and point $B$ is close enough to have mutual influence as explained in last section, then point $B$ will shift toward $A$ and the angle $A C B$ will look perceptually smaller instead of larger. This would result in the negative illusion as observed. Also, as pointed out by Walker (1973), the proposed lateral inhibition effect would also produced the negative illusion,

\section{Effect of Rotation}

Many researchers (For example, Obonai, 1931; Leibowitz \& Toffey, 1966; Weintraub \& Krantz, 1971; Bouma \& Andriessen, 1970; Green \& Hoyle, 1964) have found that the rotation of the Poggendorff figure has great influence on illu- sion; the magnitude of illusion is significantly less when the inclined line is near either horizontal or vertical. This effect is understandable, because the vertical and horizontal direction are two strong standards we can employ during our daily life. The physiological system in our inner ear maintains our sense of body orientation and we are constantly aware of the horizontal and vertical direction. These directions are two very strong references we can use and are thus less susceptible to be deviated by other inducing lines. Mathematically, this effect of rotation can be represented by a term $(A+B \sin 2 \phi)$, where $\phi$ is the angle between line BC and $\mathrm{X}$ axis, $\mathrm{A}$ and $\mathrm{B}$ are constants. Thus the illusion equation including the rotation effect would be as follows:

$$
\begin{aligned}
\mathrm{I}= & (\mathrm{A}+\mathrm{B}|\sin 2 \phi|)\left[\mathrm{K}_{\mathrm{s}}-\mathrm{K}_{1}+\mathrm{K}_{\mathrm{b}}+\mathrm{K}_{\mathrm{p}}\right. \\
& \left.+\mathrm{K}_{\mathrm{r}}^{\prime}\left(1-\mathrm{e}^{-\lambda / \mathrm{r}}\right)+\mathrm{K}_{\mathrm{a}}\right] \mathrm{d} \cot \theta .
\end{aligned}
$$

Eq. (10) is consistent with the rotation data of Bouma and Andriessen (1970), Weintraub and Krantz (1971).

\section{Orientation Change of Isolated Line Segments}

Goldstein and Weintraub (1972) have proposed that the Poggendorff illusion is due not only to the angle expansion, but also due to the orientation change of the tilted line. The transversal appears to be either more horizontal or vertical, depending on which one is nearer. Earlier, Bouma and Andriessen (1968) have also shown that the tilted line without the inducing parallel lines also appears to be closer to either horizontal or vertical, the amount of variation has been shown to be proportional to $\sin 4 \phi$. Incorporating this factor to the Poggendorff illusion, the total illusion is

$$
\begin{aligned}
\mathrm{I}= & (\mathrm{A}+\mathrm{B}|\sin 2 \phi|)\left[\mathrm{K}_{\mathrm{s}}-\mathrm{K}_{1}+\mathrm{K}_{\mathrm{b}}\right. \\
& \left.+\mathrm{K}_{\mathrm{p}}+\mathrm{K}_{\mathrm{r}}\left(1-\mathrm{e}^{-\lambda / \tau}\right)+\mathrm{K}_{\mathrm{d}}\right] \\
& \times \mathrm{d} \cot \theta+\mathrm{K} \sin 4 \phi .
\end{aligned}
$$

Discussion

Poggendorff illusion seems to be a very 
simple illusion constructed with only a few straight lines. Nevertheless, it turns out to be a quite complicated illusion. There are many factors contributed to this illusion. Eq. (11) gives a unified description of these factors and can explain the result under various kind of experimental conditions, which otherwise seem to contradict to each other. For example, Farné (1970) has shown that a modified Poggendorff display (Fig. 3) containing remote contours instead of the usual pair of parallel lines can also induce illusion. Furthermore, Goldstein and Weintraub (1972) have shown that the illusion magnitude in the Farne figure increases, contrary to the conventional Poggendorff figure, when the angle between the inclined line and the vertical parallel lines increases from $45^{\circ}$ to $51^{\circ}$. They conclude that Farné figure would require a separate explanation. However, it can be shown that Eq. (11) alone is possible to explain these results. Since the Farné figure contains only remote contours, thus the optical blurring effect $K_{b}$ is eliminated. Furthermore, since the parallel lines are missing, thus there is no physical contrast for the parallel lines; however, a perceptual contrast exists. Since the perceptual contrast is much weaker than the physical contrast, thus the illusion in Farné figure is less than the conventional Poggendorff figure. Furthermore, since the remote contour only
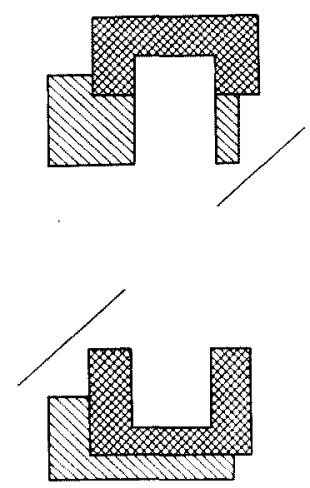

Fig. 3. A Poggendorff figure with the parallel lines replaced by contours. gives a vague perceptual contrast, the illusion is determined largely by the last term in Eq. (11), thus the illusion increases when $\theta$ changes from $45^{\circ}$ to $51^{\circ}$.

The major factors included in Eq. (11) can be classified to be: (1) physical interaction at retina level such as optical blurring. (2) neural interaction such as inhibitory effect. (3) perceptual effects such as depth processing, perceptual image distribution and variation of reference standards. While many of these factors have been separately proposed before, the concepts of perceptual image distribution and variation of reference standards are newly introduced. Furthermore, various environment factors are incorporated in a single equation so that the amount of illusion can be predicted under various experimental conditions; this equation gives a unified account of all available data, which otherwise seem to contradict to each other.

Eq. (11) contains the $\cot \theta$ component, it would seem that as $\theta$ approches zero, the amount of the illusion would approches infinity. However, it should be noted that it is very difficult to arrange an experiment with $\theta$ much less than $10^{\circ}$, this is so because $\mathbf{h}=\mathbf{d} \cot \theta$, thus as $\theta$ approches zero, $\mathrm{h}$ approches infinity and the figure will be outside the field of attention (Chiang, $1973 \mathrm{~b}$ ). With $\theta$ equal to $10^{\circ}, \mathrm{h}=5.7 \mathrm{~d}$, this seems to be the maximum length of a figure which is conveniently perceptable.

Since Eq. (11) contains many coefficients, it would be nice to obtain the relative value of these coefficients. For this purpose, systematic experiments have to be conducted with all these factors properly controlled, and the illusion is measured under various condition. So far, no experiment has yet been conducted to enable one to obtain the exact value of these coefficients in a systematic way. However, some isolated experiments have provided some estimates of these coefficients. For example, Coren (1969) has shown that the optical blurring factor $\mathrm{K}_{\mathrm{b}}$ 
contributes about $15 \%$ of the illusion. Coren (1970) has also shown the method to eliminated the possible sources of inhibitory interactions $K_{1}$ and thus can estimate the value of $K_{1}$. The effect of depth perception $K_{d}$ can be estimated by subjecting the Poggendorff figure with strong three-dimensional cues. Some modified Poggendorff figures as presented in Fig. 4 show different amount of illusion (Restle, 1969; Pressey \& den Heyer, 1968 ), this is because various influencing factors have been eliminated. These figures can be used to estimate the coefficients in Eq. (11). $\tau$ in Eq. (11) can be

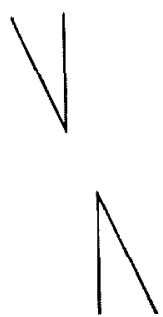

(A)

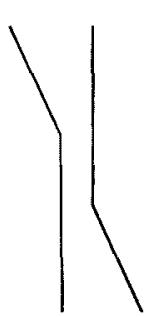

(B)

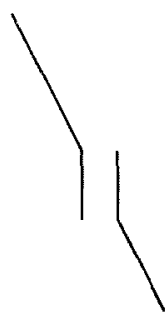

(C)
Fra. 4. Some variations of Poggendorff figure.

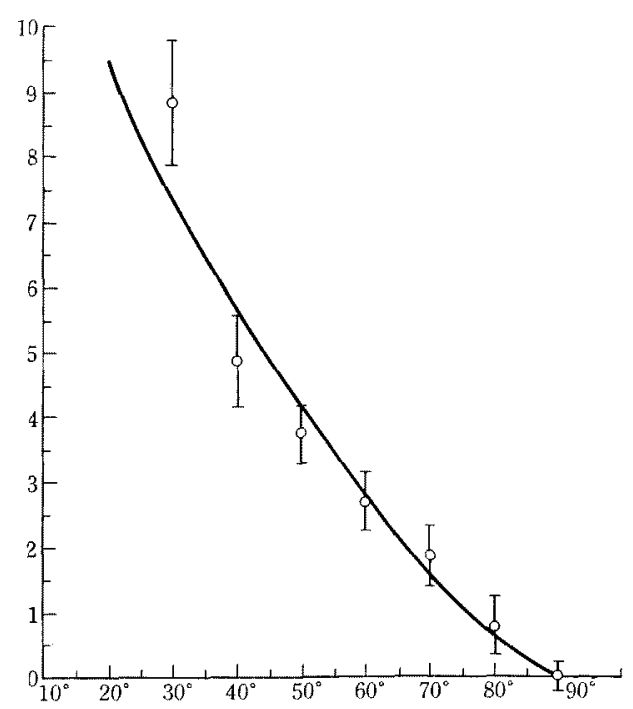

Fig. 5. Theoretical curve of $\mathrm{Eq} \cdot 11$ for $\mathrm{K}^{\prime}=1$, $\mathrm{K}=0.3, \mathrm{~B}^{\prime}=4$ together with the experimental data taken from Walker's paper. estimated from the data of Weintraub and Krantz (1971) approximately to be 0.5 .

Even though the coefficients are not known yet, we can test Eq. (11) in Fig. 5. Since $\phi=90^{\circ}-\theta$, Eq. (11) can simplify to be

$$
\begin{aligned}
\mathrm{I}= & \mathrm{K}^{\prime}\left(1+\mathrm{B}^{\prime}|\sin 2 \theta|\right) \cot \theta \\
& -\mathrm{K} \sin 4 \theta .
\end{aligned}
$$

Where $\mathrm{K}^{\prime}$ and $\mathrm{B}^{\prime}$ are new constants. $\mathrm{Eq}$. (12) is plotted in Fig. 5 for $\mathrm{K}^{\prime}=1, \mathrm{~K}=$ $0.3, \mathrm{~B}^{\prime}=4$ together with the data taken from Walker's paper (1973). It can be seen that the theoretical curve and experimental data fit pretty well. It is hoped that further experiments can be conducted systematically to obtain the exact value of the coefficients in Eq. (11).

\section{REFERENCE}

Blakemore, C., Carpenter, R., \& Georgeson, M. 1970 Lateral inhibition between orientation detectors in the human visual system. Nature, 288, 37-39.

Bouma, H., \& Andriessen, J.J. 1968 Perceived orientation of isolated line segments. Vision Research, 8, 493-507.

Bouma, H., \& Andriessen, J.J. 1970 Induced changes in the perceived orientation of line segments. Vision Research, 10, 333-349.

Chiang, C. 1968 A new theory to explain geometrical illusion produced by crossing lines. Perceptual \& Psychophysics, 3, 174-176.

Chinang, C. 1973 a Annual Report of Institute of Physics, Academia Sinica, Taipei (1972-1973).

Chindo, C. $1973 \mathrm{~b}$ A theory of the Müller-Lyer illusion, Vision Research, 13, 347-353.

Coren, S. 1969 The influence of optical aberrations on the magnitude of Poggendorff illusion. Perceptual \& Psychophysics, 6 (3), 185186.

Coren, S. 1970 Lateral inhibition and geometric illusion. Quarterly Joumal of Experimental Psychology, 22, 274-278.

Cumming, G. D. 1968 A criticism of the diffraction theory of some geometrical illusions. Perceptual \& Psychophysics, 4 (6), 375-376.

Farné, M. 1970 On the Poggendorff illusion: A note to Cumming's criticism of Chun Chiang's theory. Percepiual \& Psychophysics, 
$8,112$.

Ganz, L. 1966 Mechanism of the figural aftereffects. Psychological Review, 73, 128-150.

Gillam, M. 1971 A depth processing theory of the Poggendorff illusion. Perceptual $\&^{2} P_{s y-}$ chophysics, $10(4 \mathrm{~A}), 211-216$.

Goldstein, M., \& Weintraub, D. 1972 The parallel-less Poggendorff: Virtual contours put the illusion down but not out, Perceptual \& Psychophysics, 11 (5), 353-355.

Green, R. T., \& Hoyle, E. M. 1964 The infuence of spatial orientation on the Poggendorff illusion. Acta Psychologica, 22, 348-366.

Gregory, R. L. 1963 Distortion of visual space as inappropriate constancy scaling. Nature, 199, 678-679.

IMAI, S. 1973 Experiments on Poggendorff illusion. The fournal of Social Sciences \& Humanities (Tokyo Metropolitan University), No. 90.

Leisowitz, H., \& Torfey, S. 1966 The effect of rotation and tilt on the magnitude of the Poggendorff illusion. Vision Research, 6, 100-103.

Oronal, T. 1931 Experimentelle Untersuchungen über den Aufbau des Sehraumes. Archiv für die gesamte Psychologie, 82, 308-328.

Pressey, A. W. \& den Heyer, K. 1968 Observa- tions on Chiang's new theory of geometrical illusion. Perceptual \& Psychophysics, 4 (5), 313314.

Pressey, A. W. 1971 An extension of assimilation theory to illusions of size, area and direction. Perceptual \& Psychophysics, 9, 172-176.

Pressey, A. W. 1972 The assimilation theory of geometric illusion: An additional postulate. Perceptual $\mathcal{E}$ Psychophysics, 11, 28-30.

Pressey, A.W., \& Sweeney, O. 1972 Some puzzling results on the Poggendorff illusion. Perceptual \& Psychophysics, 12 433-437.

Restle, F. 1969 Illusion of bent line. Perceptual \& Psychophysics, 5 (5), 273-274.

Von Békésy, G. 1967 Sensory inhibition. Princeton: Princeton University Press.

Velinsky, S. 1925 Explication Physiologique de l'llusion de Poggendorff. Annee Psychologique. 26, 107-116.

WALKER, E. H. 1973 A mathematical theory of optical illusion and figural aftereffects. Perceptual \& Psychophysics, 13, 467-486.

Weintraub, D.J. \& Krantz, D. H. 1971 The Poggendorff illusion: Amputations, rotations and other perturbations. Perceptual \& Psychophysics, 10, 257-264.

(Received Sept. 5, 1974) 